Modified version

December 25, 2017

\title{
Fermionic determinant as an overlap between bosonic vacua.
}

\author{
C.D. $\mathrm{Fosco}^{a *}$ \\ and \\ F.A. Schaposnik ${ }^{b \dagger}$ \\ ${ }^{a}$ Centro Atómico Bariloche, 8400 Bariloche, Argentina \\ ${ }^{b}$ Departamento de Física, Universidad Nacional de La Plata \\ C.C. 67, 1900 La Plata, Argentina
}

\begin{abstract}
We find a representation for the determinant of a Dirac operator in an even number $D=2 n$ of Euclidean dimensions as an overlap between two different vacua, each one corresponding to a bosonic theory with a quadratic action in $2 n+1$ dimensions, with identical kinetic terms, but differing in their mass terms. This resembles the overlap representation of a fermionic determinant (although bosonic fields are used here). This representation may find applications to lattice field theory, as an alternative to other bosonized representations for Dirac determinants already proposed.
\end{abstract}

*CONICET

†Investigador CICBA, Argentina 
Based on an earlier idea of Kaplan [1], the overlap formalism [2, 3] has been proposed as a way to define fermionic chiral determinants. Its lattice implementation seems to overcome the kinematical constraints imposed by the Nielsen-Ninomiya theorem [4]. It may thus provide a suitable framework to study interesting non-perturbative phenomena in models containing chiral fermions.

In the overlap approach, the determinant of a chiral Dirac operator in $D=2 d$ dimensions is defined as the overlap, i.e., scalar product, between the Dirac vacuum states of two auxiliary Hamiltonians acting on Dirac fermions in $2 d+1$ dimensions. This method has been recently extended to the case of Dirac determinants in odd dimensions [5, 6].

The overlap between two vacua can be implemented in at least two different ways: an 'operatorial' version, based on building up the Dirac vacua by occupying all the respective negative energy states, and then computing the scalar product; and also in a path integral approach, which introduces an extra dimension into the game. The ovelap is obtained by calculating a path integral amplitude for a system whose Lagrangian has a mass that depends on the coordinate labeling points in the extra dimension (in the spirit of the domain-wall picture).

In this letter we shall provide an alternative definition for a fermionic determinant as an overlap between two bosonic vacua. The issue of bosonizing a Dirac operator, namely, writing a fermionic determinant in $D$ dimensions as a functional integral over purely bosonic fields tion [7, 8]. The approaches [7, 8] share the property of involving an infinite number of bosonic fields, although for different reasons than in the overlap approach. The infinite number of fields manifests itself as an extra dimension in [ [B], and as a (discrete) infinite number of fields in the approach of [0] Besides, they deal not with the chiral case, but rather with systems involving both chiralities, i.e., Dirac fermions, the same case we shall consider in the present work. This is, in practice, the interesting case for lattice $Q C D$. In treatments that avoid the quenched approximation, a bosonic representation for the quark determinant (which is real) may be very useful indeed. We will, in this letter, also deal with this case.

\footnotetext{
${ }^{1}$ This is all what is meant by 'bosonization' here. Note that this meaning is quite different to the one used, for example, in [9].
} 
In our construction, it is convenient to write the vacuum state in the functional Schrödinger representation [10] (we shall later on discuss an operatorial representation). In the Schrödinger representation, the "wavefunction" that describes the vacuum state for a real scalar field $\varphi$ with a quadratic action is, in the "coordinate" basis, a vacuum functional $\left\langle\varphi \mid \Psi_{\Omega}\right\rangle=\Psi_{\Omega}(\varphi)$, of the following kind

$$
\Psi_{\Omega}(\varphi)=\operatorname{det}^{\frac{1}{4}}\left(\frac{\Omega}{\pi}\right) \exp \left(-\frac{1}{2} \varphi \Omega \varphi\right)
$$

where we are using a shorthand notation (similar to the one of [12]), such that

$$
\varphi \Omega \varphi \equiv \int d^{D} x d^{D} y \varphi(x) \Omega(x, y) \varphi(y)
$$

and $\Omega(x, y)$ is a real, symmetric, and definite positive kernel.

In the coordinate basis, all the wavefunctions depend on the field configuration $\varphi(x)$, where $x$ labels the $D$ 'spatial' coordinates in a $D+1$ dimensional spacetime. Thus the scalar product between two states $\Phi_{1}, \Phi_{2}$ is defined by a functional integral

$$
\left\langle\Phi_{1} \mid \Phi_{2}\right\rangle=\int \mathcal{D} \varphi \Phi_{1}^{*}(\varphi) \Phi_{2}(\varphi)
$$

where the integration measure in (3) is formally defined as $\mathcal{D} \varphi \equiv \prod_{x} d \varphi_{x}$ with $x$ in the $D$ dimensional space.

The functional determinant factor in (11) is introduced in order to normalize this wavefunction

$$
\left\langle\Psi_{\Omega} \mid \Psi_{\Omega}\right\rangle=1
$$

This vacuum state can be thought of as the ground state for a (second quantized) Hamiltonian $\mathcal{H}(\pi, \varphi)$

$$
\mathcal{H}(\pi, \varphi)=\frac{1}{2}\left[\int d^{D} x \pi^{2}(x)+\int d^{D} x \int d^{D} y \varphi(x) \Omega^{2}(x, y) \varphi(y)\right]
$$

with $\pi(x)=\delta / i \delta \varphi(x)$ the momentum operator, and $\varphi$ acting multiplicatively. Following notation (2), we write $\Omega^{2}(x, y)=\int d^{D} z \Omega(x, z) \Omega(z, y)$. As an example, one has for a free real scalar field $\Omega^{2}(x, y)=\left(-\nabla_{x}^{2}+m^{2}\right) \delta(x-y)$.

Let us now consider the overlap between two vacuum states, $\Psi_{\Omega_{1}}(\varphi)$, $\Psi_{\Omega_{2}}(\varphi)$, corresponding to two Hamiltonians, differing just in their (commuting) kernels $\Omega_{1}, \Omega_{2}$. Performing a functional Gaussian integration, and 
rearranging terms, the overlap $\left\langle\Psi_{\Omega_{1}} \mid \Psi_{\Omega_{2}}\right\rangle$ yields

$$
\begin{gathered}
\left\langle\Psi_{\Omega_{1}} \mid \Psi_{\Omega_{2}}\right\rangle=\operatorname{det}^{\frac{1}{4}}\left(\frac{\Omega_{1} \Omega_{2}}{\pi^{2}}\right) \operatorname{det}^{-\frac{1}{2}}\left(\frac{\Omega_{1}+\Omega_{2}}{2 \pi}\right) \\
=\operatorname{det}^{-\frac{1}{4}}\left[\frac{1}{4}\left(\frac{\Omega_{1}}{\Omega_{2}}+\frac{\Omega_{2}}{\Omega_{1}}+2\right)\right] .
\end{gathered}
$$

Or, by defining $\mathcal{O} \equiv \frac{\Omega_{1}}{\Omega_{2}}$,

$$
\left\langle\Psi_{\Omega_{1}} \mid \Psi_{\Omega_{2}}\right\rangle=\operatorname{det}^{\frac{1}{4}}[f(\mathcal{O})]
$$

where

$$
f(x)=\frac{x}{(1+x)^{2}},
$$

and we have ignored (and so will do in what follows) irrelevant constant factors. Determinants are supposed to be adequately regularized

so that all usual properties (like $\operatorname{det}(A B)=\operatorname{det} A \cdot \operatorname{det} B))$ hold. The symmetry $f(x)=f\left(\frac{1}{x}\right)$ is a consequence of the symmetry of the overlap (6) under $1 \leftrightarrow 2$.

The generalization to the case of a complex scalar field, endowed with a complex hermitean definite positive covariance $\Omega$, is straightforward. One obtains, instead of (7), the result

$$
\left\langle\Psi_{\Omega_{1}} \mid \Psi_{\Omega_{2}}\right\rangle=\operatorname{det}^{\frac{1}{2}}[f(\mathcal{O})] .
$$

This of course generalizes to the case of $N_{f}$ complex flavours. The results implies just to a change of the exponent of the determinant in the form (9) to $\operatorname{det}^{\frac{N_{f}}{2}}[f(\mathcal{O})]$.

As usual in similar approaches, we shall assume that the operators $\Omega_{1}$, $\Omega_{2}$ have been regularized (on the lattice, for example), what makes them bounded. We shall also assume there is a non-vanishing gap in their spectra. Then their ratio $\mathcal{O}$ is also regularized. Moreover, we will let it depend on a mass parameter, say $M$, such that

$$
M \rightarrow \infty \Rightarrow\|\mathcal{O}(M)\|<<1
$$

then

$$
\left\langle\Psi_{\Omega_{1}} \mid \Psi_{\Omega_{2}}\right\rangle \sim \operatorname{det}^{\frac{1}{2}}\left[\frac{\Omega_{1}}{\Omega_{2}}\right] \text { when } M \rightarrow \infty
$$


To deal with the specific example of Dirac operators, we will consider the case of $\Omega_{1}$ and $\Omega_{2}$ such that

$$
\Omega_{1}^{2}=-\not D^{2}+m^{2}, \Omega_{2}^{2}=-\not D^{2}+M^{2}
$$

where $\not D=\gamma_{\mu} D_{\mu}, D_{\mu}=\partial_{\mu}+A_{\mu}$, and $A_{\mu}$ is an (Abelian or non-Abelian) gauge connection. To apply the result (11) to the present case, we need to assume that $\not D$ is regularized (in the lattice, say). Then, keeping the regulator cutoff finite, and letting $M>>m, M>>\|\not D\|$, we have, for the case of $N_{f}$ flavours,

$$
\left\langle\Psi_{\Omega_{1}} \mid \Psi_{\Omega_{2}}\right\rangle \sim \operatorname{det}^{\frac{N_{f}}{4}}\left[\frac{-\not D^{2}+m^{2}}{-\not D^{2}+M^{2}}\right] \sim \operatorname{det}^{\frac{N_{f}}{4}}\left[\frac{-\not D^{2}+m^{2}}{M^{2}}\right] .
$$

Note that the regulating cutoff must be kept finite when taking this large $M$ limit, and indeed, $M$ should be in fact bigger than the cutoff. Of course, if we normalize by dividing (13) by the same object evaluated for zero external field $(A=0)$, we obtain

$$
\frac{\left\langle\Psi_{\Omega_{1}} \mid \Psi_{\Omega_{2}}\right\rangle}{\left.\left\langle\Psi_{\Omega_{1}} \mid \Psi_{\Omega_{2}}\right\rangle\right|_{A=0}} \sim \operatorname{det}^{\frac{N_{f}}{4}}\left[\frac{-\not D^{2}+m^{2}}{-\not \partial^{2}+m^{2}}\right]
$$

Of course, the number $N_{f}$ of bosonic flavours in the overlap must be adjusted, depending on the actual number of fermionic flavours $N_{F}$ considered. It is evident that we can only consider an even $N_{f}$, and moreover $N_{f}=2 N_{F}$. Then,we shall write our main result as

$$
\operatorname{det}^{\frac{N_{F}}{2}}\left[\frac{-\not D^{2}+m^{2}}{-\not \partial^{2}+m^{2}}\right] \sim \frac{\left\langle\Psi_{\Omega_{1}} \mid \Psi_{\Omega_{2}}\right\rangle}{\left.\left\langle\Psi_{\Omega_{1}} \mid \Psi_{\Omega_{2}}\right\rangle\right|_{A=0}}
$$

We have then been able to write the Dirac operator determinant for a $D$ dimensional theory of Dirac fermions with $N_{F}$ flavour in terms of the ratio of two overlaps between vacuum states in a $D+1$ dimensional bosonic theory. Note that since we are working with a Dirac operator in $D=2 n$ dimensions, one has the identity $\operatorname{det}\left(-\not D^{2}+m^{2}\right)=\operatorname{det}(\not D+m)^{2}$. Then, our overlap formula gives in fact the value for $|\operatorname{det}(\not D+m)|$. We see that our approach, as Slavnov's and Luscher's, only works in even dimensions. The reason is that only in even dimensions is the Dirac determinant real. The extension of 
our method to odd dimensions would imply the necessity of defining vacuum functionals for non-Hermitian Hamiltonians.

There is a striking similarity between this case, for a finite $M$ (see equation (7)), and what the standard overlap yields for the modulus of a chiral determinant: $|\langle A+\mid A-\rangle|=\operatorname{det}^{\frac{1}{2}}\left(\frac{C^{\dagger} C}{C^{\dagger} C+M^{2}}\right)$, where $C$ is the chiral Dirac operator [13].

For the concrete example of the Dirac operator in $D=2$ dimensions, we may even interpret the ratio $\mathcal{O}$ as a Pauli-Villars regularization for the operator .. $/ \Omega_{1}=\sqrt{-\not D^{2}+m^{2}}$, since just one regulator field suffices (the contribution of this regulator is of course $\Omega_{2}$ ). We still assume a lattice regularization. Then the overlap for this case is doubly regulated, when one takes the large- $M$ limit (in particular, $M>>1 / a$, where $a$ is the lattice spacing), one is removing the Pauli-Villars regulator, and at the same time approaching the determinant. The latter is still regulated since $a$ is kept finite.

We will also give an operatorial construction of the overlap (6) between the two vacuum states, which avoids the use of the Schrödinger representation. We need to introduce suitable creation and annihilation operators. As the Hamiltonian is quadratic, we shall first consider the case of one simple harmonic oscillator, and then generalize to the case of interest, which contains an infinite collection of decoupled harmonic modes. For a harmonic oscillator, the analogous of (6) would be to evaluate the scalar product between to vacua, each one corresponding to a Hamiltonian with a given frequency $\omega$. Namely, we need to evaluate (using operatorial methods) the object $\left\langle\Psi_{\omega_{1}} \mid \Psi_{\omega_{2}}\right\rangle$ where $\left|\Psi_{\omega_{1}}\right\rangle$ and $\left|\Psi_{\omega_{2}}\right\rangle$ are the ground states of $H_{1}=\frac{1}{2}\left(p^{2}+\omega_{1}^{2} q^{2}\right)$ and $H_{2}=\frac{1}{2}\left(p^{2}+\omega_{2}^{2} q^{2}\right)$, respectively. It is possible to show that, if we define the corresponding two sets of creation and annihilation operators $a_{i}, a_{i}^{\dagger}$, $i=1,2$, they will be related by the Bogoliubov transformation. This transformation is exactly of the kind that appears when defining "squeezed states" [14]:

$$
\begin{aligned}
& a_{1}=\cosh \theta a_{2}+\sinh \theta a_{2}^{\dagger}=U_{\theta} a_{2} U_{\theta}^{\dagger} \\
& a_{1}^{\dagger}=\sinh \theta a_{2}+\cosh \theta a_{2}^{\dagger}=U_{\theta} a_{2}^{\dagger} U_{\theta}^{\dagger}
\end{aligned}
$$

where

$$
\cosh \theta=\frac{1}{2}\left(\sqrt{\frac{\omega_{1}}{\omega_{2}}}+\sqrt{\frac{\omega_{2}}{\omega_{1}}}\right)
$$




$$
\sinh \theta=\frac{1}{2}\left(\sqrt{\frac{\omega_{1}}{\omega_{2}}}-\sqrt{\frac{\omega_{2}}{\omega_{1}}}\right)
$$

and

$$
U_{\theta}=\exp \left[\frac{1}{2} \theta\left(a_{2}^{2}-\left(a_{2}^{\dagger}\right)^{2}\right)\right]
$$

¿From this it follows that we can write one vacuum in terms of the other as $\left.\Psi_{\omega_{1}}\right\rangle=U_{\theta}\left|\Psi_{\omega_{2}}\right\rangle$. It is evident that $\left|\Psi_{\omega_{1}}\right\rangle$, when represented in the Hilbert space built with $a_{2}^{\dagger}$ acting on $\left|\Psi_{\omega_{2}}\right\rangle$, will be a linear combination of states containing an even number of excitations. After some algebra, this can be put more explicitly as follows [14

$$
\left|\Psi_{\omega_{1}}\right\rangle=e^{-\frac{1}{2} \ln \cosh \theta} e^{-\frac{1}{2}\left(a^{\dagger}\right)^{2} \tanh \theta}\left|\Psi_{\omega_{2}}\right\rangle
$$

¿From (19), we obtain for the overlap

$$
\left\langle\Psi_{\omega_{1}} \mid \Psi_{\omega_{2}}\right\rangle=e^{-\frac{1}{2} \ln \cosh \theta}=\left[2 f\left(\frac{\omega_{1}}{\omega_{2}}\right)\right]^{\frac{1}{4}}
$$

with $f$ as defined in (8). This is clearly the equivalent of (7) for the case of a $0+1$ field theory. But the generalization to the $D+1$ case is trivial because the Hamiltonians are quadratic, and the system is brought to an infinite collection of uncoupled harmonic oscillators by using operators that create or destroy particles occupying states that are eigenmodes of the kernels $\Omega_{1}^{2}$ and $\Omega_{2}^{2}$ (they are Hermitian, and differ by an operator proportional to the identity). These, of course, are the usual plane waves when $\Omega^{2}(x, y)=$ $\left(-\nabla_{x}^{2}+m^{2}\right) \delta(x-y)$.

Defining thus the eigenmodes $g_{k}(x)$ by

$$
\int d^{D} y \Omega_{1}^{2}(x, y) g_{k}(y)=\lambda_{k}^{2} g_{k}(x), \quad \int d^{D} y \Omega_{2}^{2}(x, y) g_{k}(y)=\lambda_{k}^{\prime 2} g_{k}(x)
$$

we obviously have $\lambda_{k}^{2}-\lambda_{k}^{\prime 2}=M^{2}-m^{2}$. Then we can apply (20) to each mode, which now has frequency $\lambda_{k}^{2}\left(\right.$ or $\left.\lambda_{k}^{\prime 2}\right)$. This yields

$$
\left\langle\Psi_{\Omega_{1}} \mid \Psi_{\Omega_{2}}\right\rangle=\prod_{\lambda_{k}}\left[f\left(\frac{\lambda_{k}^{2}}{\lambda_{k}^{\prime 2}}\right)\right]^{\frac{1}{4}}=\operatorname{det}^{\frac{1}{4}}[f(\mathcal{O})]
$$


in agreement with (7).

We conclude mentioning that our approach shares with the overlap definition and with the proposals of [8, [] the property of involving an infinite number of fields, here manifested in the use of a system living in $D+1$ dimensions. Our method uses bosons rather than fermions, what means that the $D+1$ dimensional theory would be rather unphysical, as in $[7$, $8 \pi$, in the sense that the action attributed to the bosons would be quite exotic. It may however, be a useful technique for lattice simulations, even in the framework of the Schrödinger functional approach, which can be implemented on the lattice [11]. This because our representation would allow to include the effect of fermion loops without having to simulate fermions, a well known stumbling block for numerical simulations. Of course, one may also attempt to solve for the vacuum functional analyticaly, for example by variational techniques, and then calculate the overlap.

Acknowledgements: F.A.S. is partially suported by CICBA and CONICET, Argentina and a Commission of the European Communities contract No:C11*_CT93-0315. C.D.F. is supported by CONICET. We acknowledge Professors H. Neuberger and S. Randjbar-Daemi for comments on a preliminary version of this letter.

\section{References}

[1] D. B. Kaplan, Phys. Lett. B288 342 (1992).

For a review of Kaplan's formulation, see: K. Jansen, Phys. Rept. 273: 1-54 (1996).

[2] R. Narayanan and H. Neuberger, Nucl. Phys. B443:305-385 (1995). See also: R. Narayanan, H. Neuberger and P. Vranas, Nucl. Phys. Proc. Suppl.47: 596-598 (1996); R. Narayanan and H. Neuberger, Nucl. Phys. Proc. Suppl. 47: 591-595 (1996); H. Neuberger, hep-lat/9511001.

[3] S. Randjbar-Daemi and J. Strathdee, Nucl. Phys. B443:386-416 (1995);

S. Randjbar-Daemi and J. Strathdee, Nucl. Phys. B466: 335-360 (1996).

[4] L. H. Karsten, Phys. Lett. 104B, 315 (1981);

L. H. Karsten and J. Smit, Nucl. Phys B183, 103 (1981); 
H. B. Nielsen and M. Ninomiya, Nucl. Phys B185, 20 (1981); Nucl. Phys B193, 173 (1981); Phys. Lett. 105B, 219 (1981). For a review, see: Y. Shamir, Nucl. Phys. Proc. Suppl. 47: 212-227 (1996).

[5] R. Narayanan and J. Nishimura, Nucl. Phys. B508: 371-387 (1997).

[6] Y. Kikukawa and H. Neuberger, e-Print Archive: hep-lat/9707016.

[7] M. Luscher, Nucl. Phys. B418: 637-648 (1994).

[8] A.A. Slavnov, Phys. Lett.B366:253-260 (1996);

A.A. Slavnov, Phys. Lett.B388:147-153 (1996);

A.A. Slavnov, e-Print Archive: hep-th/9611154;

T.D. Bakeev, M.I. Polikarpov, A.A. Slavnov and A.I. Veselov, e-Print Archive: hep-lat/9702002.

[9] C. P. Burgess and F. Quevedo, Nucl. Phys. B421 (1994) 373;

C. P. Burgess and F. Quevedo, Phys. Lett. B 329 (1994) 457;

C. Burgess, C.A. Lütken and F. Quevedo, Phys. Lett.B 336 (1994) 18.

[10] For an excelent review, see for example: R. Jackiw Analysis on infinite dimensional manifolds: Schrodinger representation for quantized fields, (MIT, LNS). MIT-CTP-1632, Aug 1988. Presented at Seminar on Higher Mathematics, Montreal, Canada, Jun 1988. Pub. in Brazil Summer School 1989:78-143.

[11] See, for example, M. Luscher, R. Narayanan and P. Weisz, Nucl. Phys. B384: 168-228 (1992).

[12] See, for example, J. Zinn-Justin, Quantum Field Theory and Critical Phenomena, Clarendon Press, Oxford, 1993.

[13] C.D. Fosco and R.C. Trinchero, Phys. Lett. B394: 170-178 (1997).

[14] H. Umezawa; Advanced Field Theory, AIP, New York (1993). 